\title{
Use of larvae of the wax moth Galleria mellonella as an in vivo model to study the virulence of Helicobacter pylori
}

Maria Giannouli, ${ }^{1,2}$, Anna Teresa Palatucci ${ }^{3,4}$, Valentina Rubino ${ }^{3}$, Giuseppina Ruggiero ${ }^{3}$, Marco Romano $^{5}$, Maria Triassi ${ }^{2}$, Vittorio Ricci ${ }^{* *}$ and Raffaele Zarrilli ${ }^{2,6^{*}}$

\begin{abstract}
Background: Helicobacter pylori is the first bacterium formally recognized as a carcinogen and is one of the most successful human pathogens, as over half of the world's population is colonized by the bacterium. H. pylori-induced gastroduodenal disease depends on the inflammatory response of the host and on the production of specific bacterial virulence factors. The study of Helicobacter pylori pathogenic action would greatly benefit by easy-to-use models of infection.

Results: In the present study, we examined the effectiveness of the larvae of the wax moth Galleria mellonella as a new model for $\mathrm{H}$. pylori infection. G. mellonella larvae were inoculated with bacterial suspensions or broth culture filtrates from either different wild-type $\mathrm{H}$. pylori strains or their mutants defective in specific virulence determinants, such as VacA, CagA, CagE, the whole pathogenicity island (PAI) cag, urease, and gamma-glutamyl transpeptidase (GGT). We also tested purified VacA cytotoxin. Survival curves were plotted using the Kaplan-Meier method and $\mathrm{LD}_{50}$ lethal doses were calculated. Viable bacteria in the hemocoel were counted at different time points post-infection, while apoptosis in larval hemocytes was evaluated by annexin $V$ staining. We found that wild-type and mutant $H$. pylori strains were able to survive and replicate in G. mellonella larvae which underwent death rapidly after infection. H. pylori mutant strains defective in either VacA, or CagA, or CagE, or cag PAl, or urease, but not GGT-defective mutants, were less virulent than the respective parental strain. Broth culture filtrates from wild-type strains G27 and 60190 and their mutants replicated the effects observed using their respective bacterial suspension. Also, purified VacA cytotoxin was able to kill the larvae. The killing of larvae always correlated with the induction of apoptosis in hemocytes.
\end{abstract}

Conclusions: G. mellonella larvae are susceptible to H. pylori infection and may represent an easy to use in vivo model to identify virulence factors and pathogenic mechanisms of $H$. pylori. The experimental model described can be useful to screen a large number of clinical $H$. pylori strain and to correlate virulence of $H$. pylori strains with patients' disease status.

Keywords: Helicobacter pylori, Virulence factors, Galleria mellonella, Infection, Apoptosis

\footnotetext{
*Correspondence: vricci@unipv.it; rafzarri@unina.it

'Department of Molecular Medicine, Human Physiology Section, University of

Pavia Medical School, Pavia, Italy

${ }^{2}$ Department of Public Health, Hygiene Section, University of Naples

"Federico II", Naples, Italy

Full list of author information is available at the end of the article
} 


\section{Background}

Helicobacter pylori is a gram-negative, microaerophilic bacterium that colonizes approximately $50 \%$ of the world's population. H. pylori infection causes chronic gastritis, which is asymptomatic in the majority of carriers but may evolve into more severe disease, such as atrophic gastritis, gastric and duodenal ulcers, mucosa-associated lymphoid tissue lymphoma and gastric adenocarcinoma [1,2]. $H$. pylori-induced gastroduodenal disease depends on the inflammatory response of the host and on the production of specific bacterial virulence factors, such as urease, the vacuolating cytotoxin VacA, gamma-glutamyl transpeptidase (GGT), and a 40-kbp pathogenicity island (cag PAI) encoding the $120-145 \mathrm{kDa}$ immunodominant protein cytotoxin-associated gene A (CagA) as well as a type IV secretion system that injects CagA into the host cell [1-9]. The availability of a large number of genome sequences of $H$. pylori strains isolated from asymptomatic individuals and patients with gastric cancer, peptic ulcer disease, or gastritis provides the opportunity to identify novel virulence factors and mechanisms of diseases [10-12].

In vitro models of monolayers of gastric mucosal cells, either primary cultures or gastric adenocarcinoma cell lines, primary cultures of monocytes or lymphocytes have been extensively used to study $H$. pylori pathogenesis but have not been able to reproduce completely clinical outcomes associated with $H$. pylori infection [6,13-15]. Moreover, rodent models of wild-type mice, knock-out or transgenic mice and mongolian gerbils have been used to reproduce $H$. pylori persistent infection and disease [16-18]. However, these mammalian models are very expensive and time-consuming because they require specific animal facilities not widely accessible to all research groups, a large number of animals in order to obtain statistically significant results, and a formal approval by the local Ethics Committee.

Invertebrate hosts, such as nematodes or insects, can be used as alternative models of infection. Caenorhabditis elegans has been used as an infection model for a diverse range of bacterial and fungal pathogens $[19,20]$. However, C. elegans cannot survive at $37^{\circ} \mathrm{C}$ and lacks functional homologues of cellular components of the mammalian immune system, such as specialized phagocytic cells [21]. Models of infection based on insects, such as Drosophila melanogaster and Galleria mellonella (wax moth) larvae offer the advantage that they can survive at $37^{\circ} \mathrm{C}$. For example, a transgenic Drosophila model with inducible CagA expression has been used to study the signal transduction pathways activated by CagA $[22,23]$. In addition, insects possess specialized phagocytic cells, also known as hemocytes [21], which resemble mammalian phagocytes because they are able to engulf pathogens and kill them by using antimicrobial peptides and reactive oxygen species through proteins homologous to the NADPH oxidase complex of human neutrophils [24]. Moreover, genes that are known to mediate recognition of pathogenassociated molecular patterns, such as at least three different toll-like receptors and the transcription factor nuclear factor- $\mathrm{kB}(\mathrm{NFkB})$, and apoptosis-related signaling, such as caspases $-1,-3,-4$, and -6 , are expressed in G. mellonella larvae [25,26]. Although G. mellonella does not reproduce all aspects of mammalian infection, their larvae are increasingly used as mini-hosts to study pathogenesis and virulence factors of several bacterial and fungal human pathogen for the following advantages: i) low overall costs of breeding large numbers of larvae and worldwide commercial availability; ii) adaptation to human physiological temperature $\left(37^{\circ} \mathrm{C}\right)$; iii) presence of a well-characterized phagocytic system; iv) availability of a comprehensive transcriptome and immune gene repertoire [21,24-26]. G. mellonella larvae were shown to be susceptible to infection with several pathogens, including Pseudomonas aeruginosa [27], Staphylococcus aureus [28], Francisella tularensis [29], Burkholderia mallei [30], Acinetobacter baumannii [31], Klebsiella pneumoniae [32], Cryptococcus neoformans [33], Candida albicans [34] and Campylobacter jejuni [35]. Also, C. jejuni bacteria have been observed in the haemocoel and gut of infected larvae, and have been demonstrated to induce damage to the midgut [36].

In this study, we demonstrate that G. mellonella is susceptible to infection with $H$. pylori and may represent a valuable model to identify virulence factors and pathogenic mechanisms of $H$. pylori.

\section{Methods}

\section{Bacterial strains and growth conditions}

A total of eleven $H$. pylori strains were included in this study. In particular, we used: a) the wild-type $H$. pylori strain G27 $\left(\mathrm{VacA}^{+} / \mathrm{cagPAI}^{+} /\right.$urease $\left.^{+}\right)$and its isogenic mutants in which the $\operatorname{cagA}(\mathrm{G} 27 \Delta \operatorname{cag} A)$ or $\operatorname{cag} E$ $(\mathrm{G} 27 \Delta c a g E)$ gene or the entire cagPAI (G27 $\Delta$ cagPAI) were disrupted by insertional mutagenesis [3,37]; b) the wild-type $H$. pylori strain 60190 (ATCC 49503; $\mathrm{VacA}+\mathrm{s} 1 / \mathrm{i} 1 / \mathrm{m} 1 /$ cagPAI $^{+} /$urease $\left.^{+}\right)$and its isogenic mutants in which vacA (60190 $\Delta v a c A)$, or $\operatorname{cagA}(60190 \Delta c a g A)$, or $\operatorname{cagE}(60190 \Delta c a g E)$ were disrupted by insertional mutagenesis $[38,39]$ as well as its urease-negative spontaneous mutant urease (60190 Urease-negative) [40]; c) the mouse-adapted $H$. pylori strain M5 and its GGT-defective isogenic mutant (M5ggt::aph) in which ggt was disrupted by insertional mutagenesis [8].

Bacteria were cultured on Columbia agar supplemented with 10\% defibrinated horse blood, 1\% Vitox and Skirrow's supplement under microaerophilic conditions in anaerobic jars with microaerobic System CampyGen (all from Oxoid, Milan, Italy) at $37^{\circ} \mathrm{C}$ for 3 days. 


\section{Preparation of broth culture filtrates (BCFs)}

BCFs were prepared as previously described [41,42]. Briefly, bacteria were grown in Brucella broth medium supplemented with 1\% Vitox and Skirrow as well as 5\% heat-inactivated fetal calf serum (FCS; Sigma-Aldrich, Milan, Italy) in anaerobic jars with microaerobic System CampyGen with gentle shaking (150 oscillations/min) for $24-48 \mathrm{~h}$ at $37^{\circ} \mathrm{C}$. When bacterial suspensions reached 1.0 optical density units at $450 \mathrm{~nm}$ (corresponding to a bacterial concentration of $5 \times 10^{8}$ colony-forming units (CFUs/ml), bacteria were removed by centrifugation $(12,000 \mathrm{~g}$ for $15 \mathrm{~min})$, and the supernatants were sterilized by filtering through a $0.22-\mu \mathrm{m}$-pore-size cellulose acetate filter (Sartorius Minisart SM 16534, SigmaAldrich) to obtain BCFs.

\section{Purification and use of VacA toxin}

VacA (s1/m1 genotype) was purified by ammonium sulphate precipitation and gel filtration chromatography from wild-type H. pylori 60190 strain grown in Brucella broth in which foetal calf serum was replaced by $0.2 \%$ $\beta$-cyclodextrins (Sigma-Aldrich) [43,44]. Purified VacA was stored in melting ice and, immediately before use on G. mellonella larvae, was activated or not by dropwise acidification to $\mathrm{pH} 3.0$ with $0.2 \mathrm{~N} \mathrm{HCl}$. Vacuolating activity of purified VacA was determined by means of neutral red uptake as previously described [45]. In order to compare the effects obtained with either purified VacA or $\mathrm{VacA}^{+}$ $\mathrm{BCF}$, it must be taken into account that the vacuolating power on cultured human epithelial cells of $\mathrm{VacA}^{+} \mathrm{BCF}$ prepared as above from $60190 \mathrm{H}$. pylori strain was equivalent to that exhibited by a final concentration of $1.2 \mu \mathrm{g} / \mathrm{ml}$ of activated purified VacA [42,45].

\section{G. mellonella killing assays}

To assess the virulence of $H$. pylori in vivo using the G. mellonella insect model of infection [26], caterpillars weighing between $200 \mathrm{mg}$ and $400 \mathrm{mg}$ and maintained on wood chips in the dark at $8-10^{\circ} \mathrm{C}$ were employed in all assays. No ethical approval was required for the study because there was no use of a mammalian model of infection and animal house. Briefly, bacteria were harvested from a culture by rolling a moistened swab over the plate into $1 \mathrm{ml}$ of phosphate-buffered saline (PBS) and adjusted to an $\mathrm{OD}_{450}$ of 1.0. A Hamilton syringe was used to inject $10 \mu \mathrm{l}$ aliquots of serially diluted bacterial suspensions (from $1 \times 10^{7}$ to $1 \times 10^{4}$ CFUs) or BCFs collected from $1 \times 10^{6}$ CFUs into the hemocoel via the left proleg of each larva. Bacterial colony counts on $10 \%$ blood Columbia agar plates under microaerophilic conditions were used to confirm all inocula of either bacterial suspensions or BCFs. Control larvae were either injected with $10 \mu \mathrm{l}$ of PBS in order to measure any potential lethal effects of the injection process, or not injected to measure the effects of the incubation procedure. Ten G. mellonella larvae were infected for each experimental condition, with each experiment repeated at least 3 times. After injection, larvae were incubated in petri dishes at $37^{\circ} \mathrm{C}$ in standard aerobic conditions and survival was recorded at $24 \mathrm{~h}$ intervals for $96 \mathrm{~h}$. Larvae were considered dead when they displayed no movement in response to gentle prodding with a pipette tip [31].

To determine the numbers of viable bacteria in larvae at $0,24,48$ and $72 \mathrm{~h}$ post-infection, larvae were chilled on ice for $10 \mathrm{~min}$. The bottom $2 \mathrm{~mm}$ of each larva was aseptically removed and haemocoel was drained into a sterile $1.5 \mathrm{ml}$ microcentrifuge tube. For enumeration haemocoel was serially diluted in PBS and the bacterial load per larva was quantified by enumeration of CFUs on Columbia Blood Agar plates (CBA) supplemented with $10 \%$ defibrinated horse blood, $1 \%$ Vitox and Skirrow's supplement and incubating under microaerophilic conditions in anaerobic jars with microaerobic System CampyGen (Oxoid) at $37^{\circ} \mathrm{C}$ for $48-72 \mathrm{~h}$.

\section{Flow cytometry analysis of $G$. mellonella hemocytes}

Hemocytes were prepared from hemolymph of G. mellonella larvae as described by Bergin et al. [24]. Plasma membrane asymmetry existing in living cells is lost on apoptosis and it is commonly detected with probes, like Annexin $\mathrm{V}$, interacting strongly and specifically with phosphatidylserine. In order to assess apoptosis induction on G. mellonella hemocytes, (FITC)-conjugated annexin V (Pharmingen San Diego, CA) staining has been performed as described [46]. Cells were washed in cold Annexin V buffer (10 mM HEPES, $140 \mathrm{mM} \mathrm{NaCl}, 2.5 \mathrm{mM} \mathrm{CaCl}_{2}$ ) prior to treatment with FITC-labeled Annexin V (BD, Milan, Italy) for $15 \mathrm{~min}$ at room temperature. Annexin $\mathrm{V}$ binding was evaluated by using a two laser equipped FACSCalibur apparatus and the Cell Quest analysis software (Becton Dickinson, Mountain View, CA).

\section{Statistical analyses}

All statistical analyses were carried out using GraphPad Prism version 5.04 for Windows (GraphPad Software, San Diego, CA, USA). Survival curves were plotted using the Kaplan-Meier method, and differences in survival were calculated using the log-rank test for multiple comparisons. Differences were considered statistically significant at $P<.05 . \mathrm{LD}_{50}$ values of $H$. pylori strains were calculated as described previously [47]. Briefly, GraphPad Prism was used to fit a curve to the infection data of the following form: $Y=[A+(1-A)] /[1+\exp (B-G \mathrm{x} \ln \mathrm{X})]$, where $\mathrm{X}$ is the number of viable bacterial cells injected, $\mathrm{Y}$ the fraction of larvae killed by the bacterial solution, A is the fraction of larvae killed by the control solution, and B and $\mathrm{G}$ are curve-fitting constants automatically calculated by GraphPad Prism. $\mathrm{LD}_{50}$ was calculated as the value of $\mathrm{X}$ 
that corresponds to $\mathrm{Y}=0.5$. All experiments were performed at least three times and the results were shown as means \pm SEM. Differences between mean values were tested for significance by performing either unpaired, two-tailed Student's $t$-tests or one-way ANOVA analysis followed by Tukey's multiple-comparison test, when appropriate. A $P$ value $<0.05$ was considered to be statistically significant.

\section{Results}

\section{$H$. pylori infection causes death of G. mellonella larvae}

We examined the susceptibility of $G$. mellonella to wild-type $H$. pylori strains G27, 60190 and M5, which are widely used for molecular pathogenesis studies. G. mellonella larvae were injected with $1 \times 10^{4}, 1 \times 10^{5}$, $1 \times 10^{6}$ and $1 \times 10^{7}$ CFUs of G27 and 60190 wild-type strains and incubated at $37^{\circ} \mathrm{C}$ up to $96 \mathrm{~h}$. As shown in Figure $1 \mathrm{~A}, 1 \mathrm{~B}$ and $1 \mathrm{C}, \mathrm{H}$ pylori strains $\mathrm{G} 27,60190$ and M5 caused a time- and dose-dependent death of larvae $(\mathrm{p}<0.0001)$. The percentage of surviving larvae at $24 \mathrm{~h}$ after infection with increasing doses of wild-type strains G27, 60190 and M5 ranged between 97\% and 33\%, $100 \%$ and $65 \%$, and $100 \%$ and $74 \%$, respectively. No mortality was observed in G. mellonella larvae either non-infected or PBS-injected (Figure 1A, 1B, 1C). Since the dose of $1 \times 10^{6} \mathrm{CFUs} /$ larva allowed to observe clearcut differences in virulence potential, this concentration of bacterial suspension was chosen as the optimal dosage for the subsequent virulence studies described in this paper. As shown in Figure 1D, wild-type strain G27 showed significantly increased mortality compared to wild-type strains 60190 , and M5 $(P<0.0005)$. Separately, the 50\% lethal doses $\left(\mathrm{LD}_{50}\right)$ of $\mathrm{G} 27,60190$, and M5 were determined in G. mellonella. The analysis of $\mathrm{LD}_{50}$ doses of H. pylori wild-type strains tested showed that G27 was more virulent than 60190 and M5, with $\mathrm{LD}_{50}$ values at $48 \mathrm{~h}$ of $2.78 \pm 0.4,6.1 \pm 0.4$ and $12.8 \pm 0.3 \times 10^{5} \mathrm{CFUs}$, respectively (Table 1 ). Collectively, these results demonstrate that G. mellonella is susceptible to $H$. pylori infection, in a time- and dose-dependent manner.

\section{Effect of $H$. pylori virulence factors on killing of $G$. mellonella larvae}

To identify bacterial virulence factors responsible for $H$. pylori-induced killing of G. mellonella larvae, we compared the effects of wild-type strains G27, 60190 and M5 with those of their respective mutants in selective virulence factors. The survival percentages of a group of $10 \mathrm{G}$. mellonella larvae during $72 \mathrm{~h}$ post-infection with $1 \times 10^{6} \mathrm{CFUs}$ of bacterial suspension were analyzed. As shown in Figure 2A, the wild-type strain G27 showed a statistically significant higher virulence compared with G27 $\triangle$ cagPAI, (i.e., the G27 isogenic mutant in which the entire cag PAI has been deleted), or G27 $\triangle$ cagA, or G27 $\triangle$ cagE (i.e., the
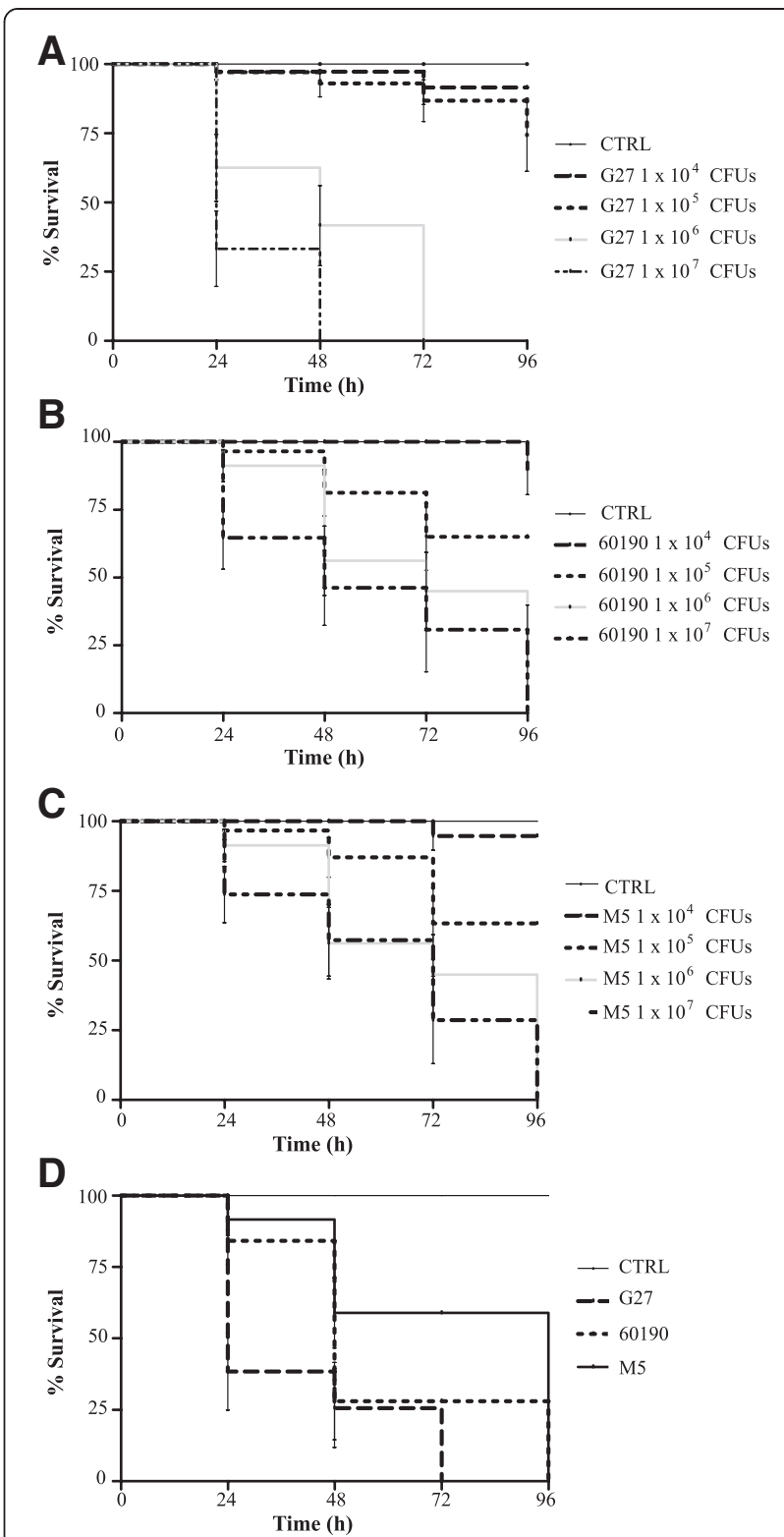

Figure 1 Survival of $G$. mellonella following infection by $H$. pylori strains. Kaplan-Meier survival curves of $G$. mellonella larvae after $24 \mathrm{~h}$-96 $\mathrm{h}$ from injection with $1 \times 10^{4}, 1 \times 10^{5}, 1 \times 10^{6}$ and $1 \times 10^{7}$ CFUs of wild type strains G27 (panel A), 60190 (panel B), M5 (panel C) are shown. Kaplan-Meier survival curves of G. mellonella larvae after 24 h-96 h from injection with $1 \times 10^{6}$ CFUs of wild-type H. pylori strains G27, 60190 and M5 (panel D) are shown. The data shown are means \pm SEM from three independent experiments recorded for $96 \mathrm{~h}$. Differences in survival were calculated using the log-rank test for multiple comparisons. Differences were considered statistically significant at $P<0.05$. PBS, phosphate-buffered saline.

G27 isogenic mutants in the effector protein CagA or in the regulatory protein CagE of the type IV secretion system, respectively). Indeed, we found $15 \%$ of larvae and no larvae alive after respectively $24 \mathrm{~h}$ and $48 \mathrm{~h}$ infection with wild type G27 strain, while 55\%-70\% and 40-45\% of larvae 
Table 1 Lethal dose $\mathbf{5 0} \%$ of $\boldsymbol{H}$. pylori strains in Galleria mellonella

\begin{tabular}{|c|c|c|}
\hline \multirow[b]{2}{*}{ Strains } & \multicolumn{2}{|c|}{ 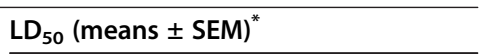 } \\
\hline & $48 \mathrm{~h}$ & $72 \mathrm{~h}$ \\
\hline $\mathrm{G} 27$ & $2.8( \pm 0.4) \times 10^{5}$ & $2.4( \pm 0.2) \times 10^{5}$ \\
\hline $\mathrm{G} 27 \Delta \mathrm{cagA}$ & & $3.1( \pm 0.04) \times 10^{6}$ \\
\hline $\mathrm{G} 27 \Delta$ cagE & & $2.4( \pm 0.06) \times 10^{6}$ \\
\hline G27 $\triangle$ cagPAl & & $2.0( \pm 0.01) \times 10^{6}$ \\
\hline 60190 & $6.1( \pm 0.4) \times 10^{5}$ & $1.4( \pm 0.04) \times 10^{6}$ \\
\hline $60190 \Delta v a c A$ & & $8.2( \pm 0.04) \times 10^{6}$ \\
\hline $60190 \Delta$ cagA & & $9.7( \pm 0.04) \times 10^{6}$ \\
\hline $60190 \Delta c a g E$ & & $9.5( \pm 0.06) \times 10^{6}$ \\
\hline 60190Urease-negative & & $8.7( \pm 0.04) \times 10^{6}$ \\
\hline M5 & $12.8( \pm 0.3) \times 10^{5}$ & $2.1( \pm 0.08) \times 10^{5}$ \\
\hline M5 ggt::aph & $12.0( \pm 0.6) \times 10^{5}$ & $1.0( \pm 0.1) \times 10^{5}$ \\
\hline
\end{tabular}

"The $\mathrm{LD}_{50}$ values were expressed in Colony Forming Units (CFUs).

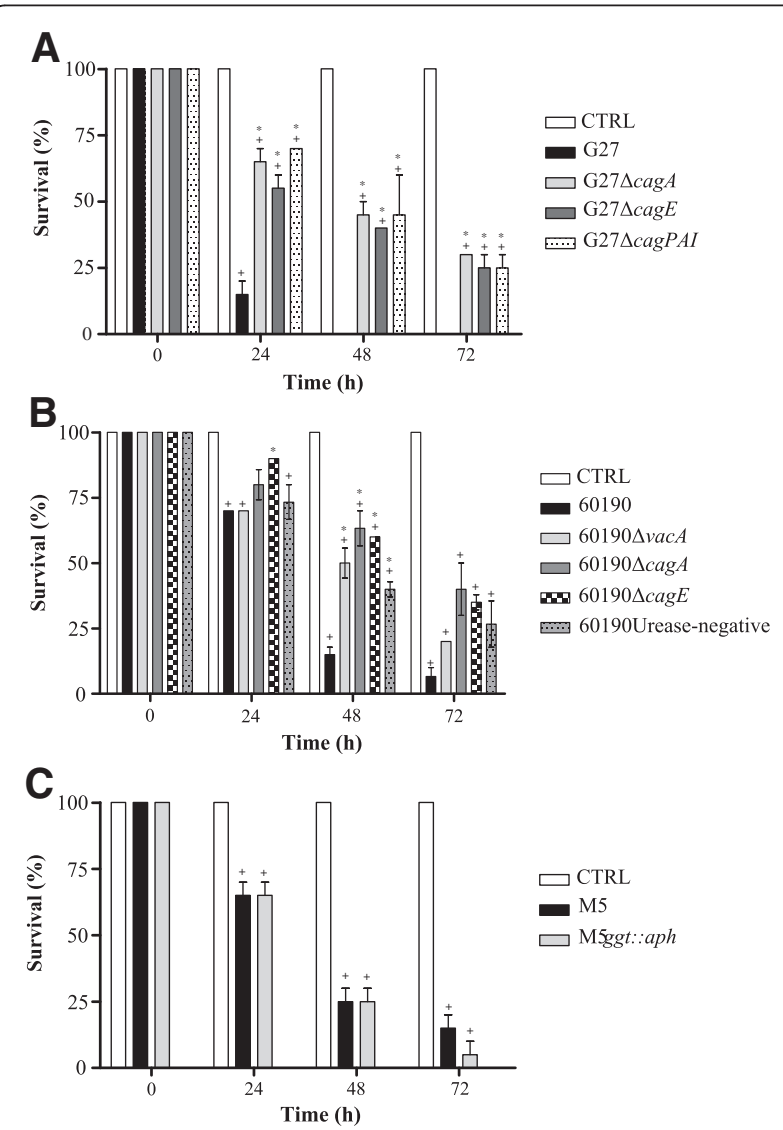

Figure 2 Ability of bacterial suspensions of $1 \times 10^{6}$ CFUs wildtype strain G27 and their mutants (panel A), wild type strain 60190 and their mutants (panel B) and wild type strain M5 and ggt-mutant (panel C) to kill G. mellonella larvae at different time points. Values represent the mean $( \pm$ SEM) of three independent experiments. $+P<0.05$ vs control (ANOVA).*; ${ }^{*} P<0.05$ vs wild-type strain (ANOVA). CTRL, control. alive after $24 \mathrm{~h}$ and $48 \mathrm{~h}$ infection with mutant strains. Moreover, the wild-type strain 60190 showed a statistically significant increased virulence compared with its isogenic mutants defective in either CagA, or CagE, or VacA as well as with its spontaneous mutant defective in urease at $48 \mathrm{~h}$ (Figure 2B). In contrast, there was no significant difference between wild type strain M5 and its GGTdefective isogenic mutant M5 ggt::aph at any time postinfection (Figure 2C).

Concordantly, $\mathrm{LD}_{50}$ values of $H$. pylori mutant strains defective in either VacA, or CagA, or CagE, cag PAI or urease- but not GGT-defective mutant, exhibited slower killing action than their respective wild type strains (Table 1). Also, all wild type strains G27, 60190 and M5 and their respective mutant strains showed a statistically significant effect on killing of G. mellonella larvae compared with control non-infected larvae $(\mathrm{p}<0.05)$ (Figure 2A-C).

Taken together, the data shown indicate that killing of larvae by $H$. pylori was at least in part dependent on the expression of a functional cag PAI, CagA, VacA cytotoxin and urease but independent of GGT.

We next determined whether death of G. mellonella was associated with the growth of $H$. pylori wild-type and mutant strains in the infected larvae. The larvae were injected with $1 \times 10^{6} \mathrm{CFUs}$ of $H$. pylori strains as described above and the number of viable bacteria within the hemolymph of G. mellonella infected larvae was determined after every $24 \mathrm{~h}$ interval. As shown in Table 2, wild-type and mutant $H$. pylori strains showed similar time-dependent increases of 1-log in the number of bacteria with no significant differences observed among strains $(P>0.05)$. The above data suggest that $H$. pylori is able to replicate in G. mellonella larvae independently of the strain virulence and that differences in killing observed between wild-type strains and mutants are not due to impaired ability of mutants to replicate into the infected host.

\section{Effect of BCFs from different wild-type $H$. pylori strains or} their isogenic mutants and of purified VacA toxin on killing of $G$. mellonella larvae

To evaluate whether the killing of G. mellonella larvae by H. pylori was dependent on a soluble bacterial virulence factor(s), the effect of BCFs from G27, 60190 and their mutants and purified VacA on killing of G. mellonella larvae was investigated. As shown in Figure 3A and 3B, BCFs from wild-type strains G27 and 60190 strains caused a time-dependent death of G. mellonella larvae with $10 \%$ and $35 \%$ of survival after $72 \mathrm{~h}$ of injection, respectively. Also, BCFs from wild-type strain G27 induced statistically higher killing of G. mellonella larvae than G27 $\Delta$ cagPAI, G27 $\triangle$ cagA and G27 $\triangle$ cagE isogenic mutant strains at $24 \mathrm{~h}$, $48 \mathrm{~h}$ and $72 \mathrm{~h}$ post injection respectively; similarly, BCFs 
Table 2 Viable count (CFUs; means \pm SEM) of $H$. pylori strains in G. mellonella at 24, 48 and 72 h post-infection

\begin{tabular}{|c|c|c|c|c|}
\hline Strains & T0 & $24 \mathrm{~h}$ & $48 \mathrm{~h}$ & $72 \mathrm{~h}$ \\
\hline G27 & $1.1( \pm 0.06) \times 10^{6}$ & $3.9( \pm 0.03) \times 10^{6}$ & $5.2( \pm 0.8) \times 10^{6}$ & $1.6( \pm 0.3) \times 10^{7}$ \\
\hline $\mathrm{G} 27 \Delta \operatorname{cagA}$ & $1.6( \pm 0.2) \times 10^{6}$ & $2.8( \pm 0.06) \times 10^{6}$ & $4.6( \pm 0.4) \times 10^{6}$ & $1.1( \pm 0.2) \times 10^{7}$ \\
\hline G27 $\operatorname{ccagE}$ & $1.0( \pm 0.1) \times 10^{6}$ & $2.2( \pm 0.04) \times 10^{6}$ & $4.0( \pm 0.6) \times 10^{6}$ & $9.2( \pm 0.3) \times 10^{6}$ \\
\hline G27 $\triangle$ cagPAl & $1.2( \pm 0.3) \times 10^{6}$ & $2.0( \pm 0.02) \times 10^{6}$ & $3.6( \pm 0.4) \times 10^{6}$ & $8.6( \pm 0.2) \times 10^{6}$ \\
\hline 60190 & $1.6( \pm 0.1) \times 10^{6}$ & $5.2( \pm 0.02) \times 10^{6}$ & $7.8( \pm 0.1) \times 10^{6}$ & $1.8( \pm 0.9) \times 10^{7}$ \\
\hline $60190 \Delta v a c A$ & $8.4( \pm 0.2) \times 10^{5}$ & $1.9( \pm 0.04) \times 10^{6}$ & $3.9( \pm 0.1) \times 10^{6}$ & $9.4( \pm 0.3) \times 10^{6}$ \\
\hline $60190 \Delta \operatorname{cag} A$ & $1.2( \pm 0.1) \times 10^{6}$ & $2.1( \pm 0.05) \times 10^{6}$ & $4.2( \pm 0.2) \times 10^{6}$ & $1.2( \pm 0.3) \times 10^{7}$ \\
\hline $60190 \Delta c a g E$ & $1.0( \pm 0.04) \times 10^{6}$ & $1.8( \pm 0.03) \times 10^{6}$ & $3.4( \pm 0.4) \times 10^{6}$ & $1.0( \pm 0.3) \times 10^{7}$ \\
\hline 60190Urease-negative & $1.4( \pm 0.06) \times 10^{6}$ & $2.6( \pm 0.2) \times 10^{6}$ & $4.9( \pm 0.4) \times 10^{6}$ & $9.8( \pm 0.2) \times 10^{6}$ \\
\hline M5 & $1.3( \pm 0.04) \times 10^{6}$ & $2.0( \pm 0.4) \times 10^{6}$ & $4.2( \pm 0.5) \times 10^{6}$ & $1.2( \pm 0.2) \times 10^{7}$ \\
\hline M5ggt::aph & $1.2( \pm 0.04) \times 10^{6}$ & $1.8( \pm 0.2) \times 10^{6}$ & $3.6( \pm 0.6) \times 10^{6}$ & $9.6( \pm 0.4) \times 10^{6}$ \\
\hline
\end{tabular}

The number of viable bacteria in infected larvae were determined as described in the Methods section and expressed in CFUs.

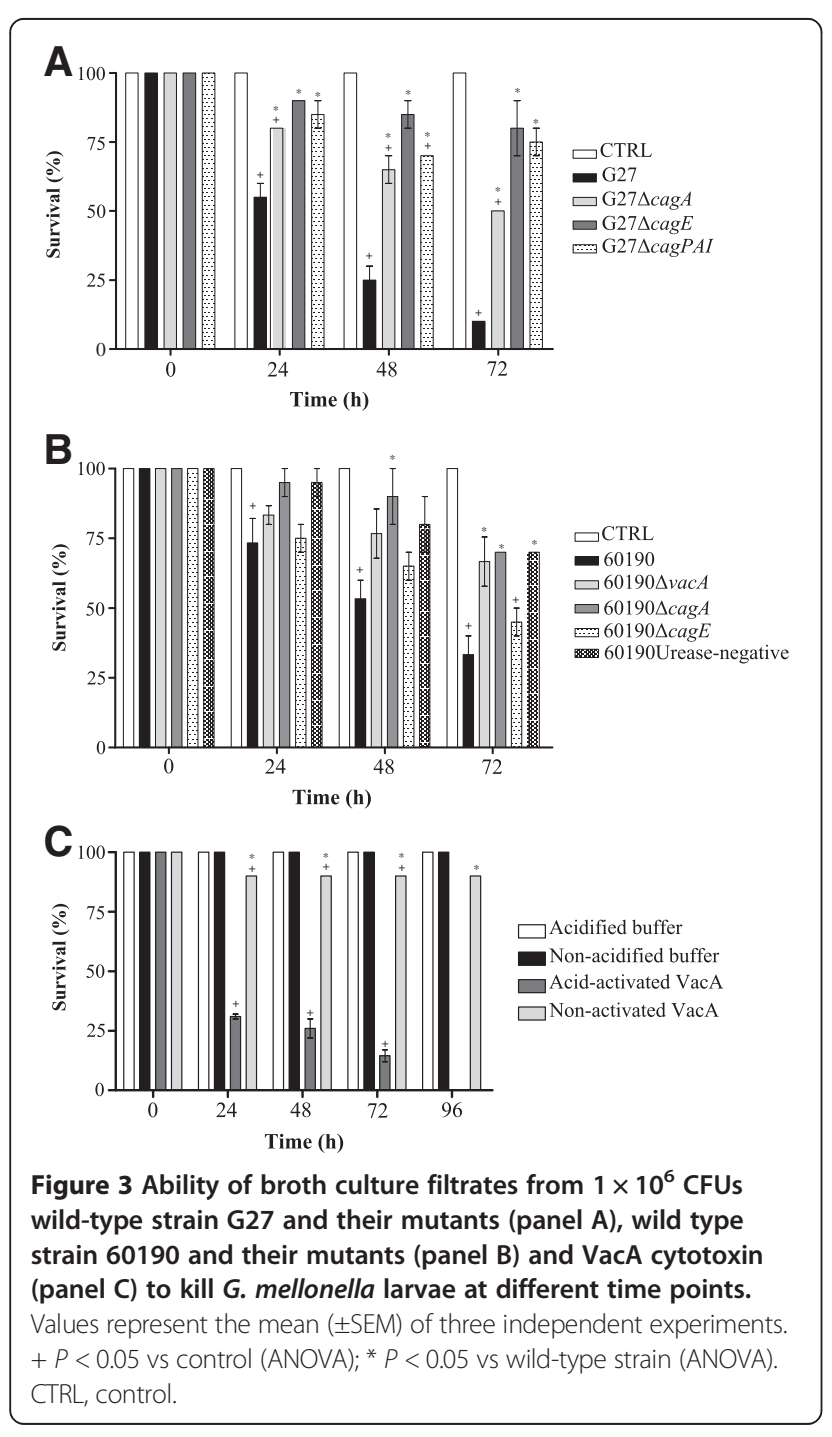

from wild-type strain 60190 induced higher killing of larvae than $60190 \Delta$ cagA at $48 \mathrm{~h}$ and $72 \mathrm{~h}$, and 60190Ureasenegative mutant at $72 \mathrm{~h}$ post-injection. No mortality was observed in the G. mellonella larvae injected with uninoculated broth filtrate taken as a control (Figure 3A and 3B). Moreover, injection of acid-activated VacA cytotoxin from 60190 H. pylori strain caused time-dependent death of larvae, with $31 \%$ survival at $24 \mathrm{~h}$ post-injection and no larvae alive at $96 \mathrm{~h}$ post-injection. On the contrary, injection of non-activated VacA caused death of $10 \%$ of larvae, injection of acidified or non-acidified control buffers caused no deaths of larvae (Figure $3 \mathrm{C}$ ). These data indicate that the effect of $H$. pylori on killing of larvae is mediated at least in part by bacterial soluble virulence factors, including VacA cytotoxin, CagA and cag PAI-encoded proteins.

H. pylori G27 and 60190 and their isogenic mutants, BCFs and VacA induce apoptosis of G. mellonella hemocytes Because it has been shown that $H$. pylori triggers the apoptotic program in different experimental systems $[2,7,9,14,23,48]$, we evaluated whether the killing of $G$. mellonella larvae by $H$. pylori might be mediated also through induction of apoptosis. To address this issue, we evaluated annexin $\mathrm{V}$ binding on hemocytes from $G$. mellonella larvae injected with bacterial suspension or BCFs of wild-type strains and mutants or purified VacA cytotoxin. As control, annexin $\mathrm{V}$ binding on uninfected hemocytes was analyzed. As shown in Figure 4A, H. pylori wild type strain G27 increased annexin V staining in G. mellonella hemocytes by 3.5 -fold compared with control uninfected larvae, while G27 $\Delta c a g E$ and G27 $\Delta$ cagPAI increased annexin $\mathrm{V}$ staining by approximately 2 -fold (p < 0.05 vs G27 strain). Concordantly, H. pylori wild type strain 60190 increased annexin V staining in G. mellonella hemocytes by approximately 2.5 -fold, while the $60190 \Delta c a g E$ demonstrated a significantly less capacity to bind the annexin (Figure 4B). These data indicate that 


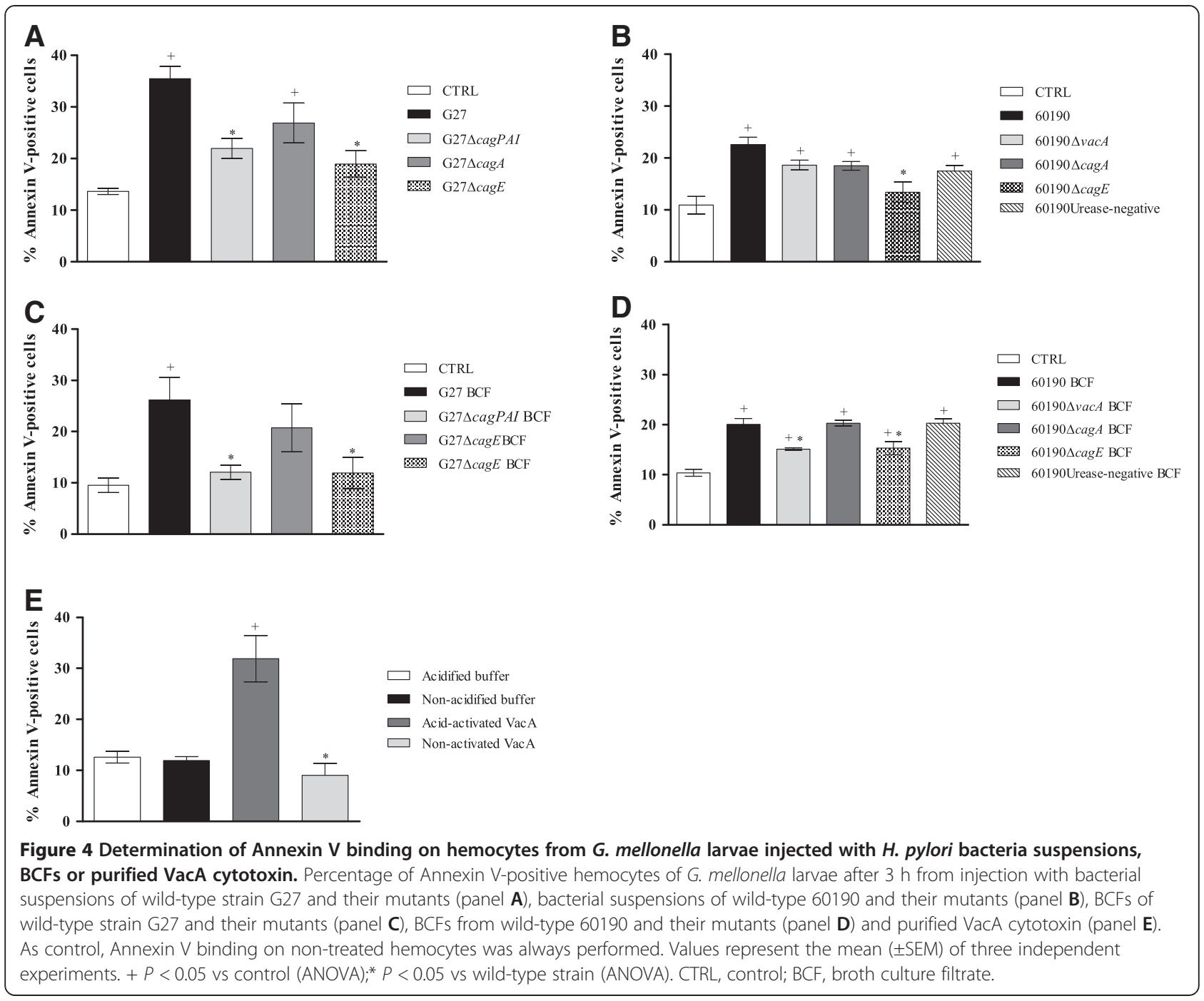

H. pylori induction of apoptosis in G. mellonella hemocytes is at least in part dependent on the expression of genes in the cag PAI.

We next evaluated the effect of soluble virulence factor (s) on apoptosis in G. mellonella hemocytes. As shown in Figure 4C, BCFs from G27 increased annexin staining by 2.5-fold, while BCFs from G27 $\triangle$ cagE and G27 $\triangle$ cagPAI demonstrated a significantly lower capacity to bind the annexin compared with BCFs from G27 strain $(\mathrm{p}<0.05)$. Also, BCFs from H. pylori wild type strain 60190 increased annexin V staining in G. mellonella hemocytes by approximately 2-fold, while the 60190 $v v a c A$ and $60190 \Delta c a g E$ mutants demonstrated a significantly lower capacity to bind the annexin compared with BCFs from 60190 strain $(P<0.05)$ (Figure 4D). Moreover, activated VacA increased annexin V staining of G. mellonella hemocytes by 3 -fold compared with non-activated VacA or control buffer or $(\mathrm{p}<0.05)$ (Figure 4D). This suggests that $H$. pylori induction of apoptosis in G. mellonella hemocytes is, at least in part, dependent on the release of soluble virulence factor(s) including VacA cytotoxin.

\section{Discussion}

In the present study, we provide evidence that the larva of the wax moth G. mellonella can be used as a new and simple infection model to study $H$. pylori virulence. We show that a panel of wild-type and mutant strains selectively defective in specific virulence factors are able to infect and kill G. mellonella larvae in a dose- and time-dependent fashion. All $H$. pylori strains analyzed are able to increase cell number by 1-log during infection of G. mellonella larvae, thus suggesting that $H$. pylori strains are able to survive and replicate in larvae. Our data also show that wild-type strain G27 is more virulent than wild-type strains 60190 and M5 and that $H$. pylori mutant strains defective in either VacA, CagA, CagE, cag PAI, or urease but not GGT-defective mutants, are less virulent than the respective parental strain. The increased ability to kill larvae of 
G27 strain compared with 60190 and M5 strains might be dependent on different genetic background and/or virulence factors different from VacA and CagA. That killing of larvae is dependent on the expression of a functional cag PAI and VacA cytotoxin is in accordance with previous data obtained in in vitro models showing that $H$. pylori-dependent epithelial cell damage and apoptosis of monocytes is dependent on VacA and cag PAI determinants [14]. Our data are also in agreement with those obtained in rodent models of $H$. pylori infection, in which inflammation and gastritis and apoptosis of monocytes and lymphocytes is dependent on the expression of both cag PAI and VacA $[17,18]$. While previous studies have shown that $H$. pylori GGT favours colonization of the gastric mucosa and more severe gastroduodenal diseases during infection in vivo $[8,9]$, here we found no difference in killing of G. mellonella larvae between the GGTdefective isogenic mutant and its parental wild-type $H$. pylori strain. This discrepancy may depend on differences between G. mellonella and rodent models of infections and/or different experimental conditions.

We also evaluated the effect of $H$. pylori soluble/ secreted virulence factors in G. mellonella larvae. In accordance with previous findings obtained in human and rodent models both in vitro and in vivo [13-18,41,44], we demonstrate that VacA, CagA and other cag PAI-encoded determinants are important soluble virulence factors of $H$. pylori strains. That soluble CagA mediates the killing of G. mellonella larvae is also in agreement with previous studies in a transgenic Drosophila model with inducible CagA expression which demonstrate that $H$. pylori CagA functions as a eukaryotic Grb2-associated binder (Gab) adaptor protein to activate the phosphatase SHP-2 and promote epithelial disruption or apoptosis through activation of the JNK signaling pathway [22,23].

Taken together, the data here presented demonstrate that $H$. pylori infection of G. mellonella larvae is a suitable model to study differences in virulence between strains. It is now well-known that $H$. pylori exhibits a high genetic and functional diversity in the cag PAI [5] as well as a high whole-genome variability among strains isolated from subjects either asymptomatic or affected by different gastroduodenal diseases [10-12]. In this respect, the infection of G. mellonella larvae may represent a useful model for the screening and the identification of virulence determinants in whole genome sequenced $H$. pylori strains.

Additional advantage provided by G. mellonella larvae infection model is the possibility to study the effect of strains and soluble virulence factors on the hemocytes, insect immune cells that are able to phagocyte bacterial and fungal cells [24] and to identify molecules responsible for immune evasion by $H$. pylori. Our data demonstrate that both $H$. pylori cells and soluble virulence factors induce apoptosis of insect hemocytes and that the effect is dependent on VacA and CagA and on the expression of a functional cag PAI. That CagA and cag PAI induce apoptosis of hemocytes is in agreement with previous studies showing that ectopic CagA expression induces apoptosis of epithelial cells of the Drosophila larval wing imaginal discs [23] and a functional cagPAI induces apoptosis of human monocytes [14]. Moreover, the effect of VacA on apoptosis of insect hemocytes is consistent with a previous study showing that VacA induces cell death in gastric epithelial cells $[15,48]$ and inhibits dendritic cell maturation in neonatally infected mice [18]. Therefore, based on the data shown herein, we have identified specific bacterial virulence factors such as CagA, cag PAI components and VacA, which are able to evade host response of insect larvae.

A limitation of this study is that the strains used in our experiments differ in origins and lab passages. This might cause the various $H$. pylori mutants have additional uncharacterized differences compared to the single wildtype parental strain used. However, we were able to compare and duplicate the effect of mutants in identical genes, i.e. cagA and cagE, in two distinct genetic backgrounds, i.e. G27 strain versus 60190 strain. This issue might more properly be addressed by comparing the killing activity in G. mellonella larvae of several datasets of wild-type and isogenic mutants displaying different genetic backgrounds.

Based on the data shown herein, we hypothesize that CagA is injected into haemocytes via a type IV secretion system. Further studies will be necessary to demonstrate this hypothesis. The NFkB pathway, which has been demonstrated to be activated by CagA and cagPAI components during apoptosis of mammalian monocytes [2] and which is expressed in G. mellonella larvae [25], should be analyzed in hemocytes following $H$. pylori infection. In addition to the effects on hemocyte apoptosis, it should be interesting to study if $H$. pylori is able to colonize and induce damage to the midgut of G. mellonella larvae, as has been recently demonstrated for $C$. jejuni [36].

The above all experiments should be the matter of a future investigation.

\section{Conclusions}

In conclusion, the model of G. mellonella larvae described herein represents a reliable and inexpensive model of $H$. pylori infection. Although the G. mellonella infection model cannot replace well-established and more "physiological" in vivo experimental models in the assessment of pathogenic mechanisms underlying $H$. pylori-related human diseases, it could be of use, and less expensive, for the evaluation of the effect of $H$. pylori virulence factors on specific cell functions.

This experimental model may reduce dependence on mammalian infection models and provide several applications for the Helicobacter research community such as 
the ability to distinguish between virulent and non-virulent H. pylori isolates, the identification of putative virulence genes through comparative genomics studies and the identification of novel molecular targets for antimicrobial therapy and vaccine development.

\section{Abbreviations \\ PAl: Pathogenicity island; GGT: Gamma-glutamyl transpeptidase; CagA: Cytotoxin-associated gene A; OD: Optical density; BCF: Broth culture filtrate; PBS: Phosphate-buffer saline solution; CFU: Colony-forming units; LD50: Lethal dose $50 \%$.}

\section{Competing interests}

The authors declare that they have no competing interests.

\section{Authors' contributions}

Conceived and designed the experiments: MG, GR, MR, VRI, RZ. Performed the experiments: MG, ATP, VRU. Analyzed the data: MG, GR, MR, MT, RZ. Wrote the manuscript: MG, VRI, RZ. All authors read and approved the final manuscript.

\section{Authors' information}

Vittorio Ricci and Raffaele Zarrilli share last authorship.

\section{Acknowledgments}

This work was supported in part by grants from the Italian Ministry for University and Research (Progetto di Ricerca di Interesse Nazionale n. 2009A37C8C_002; to Vittorio Ricci) and Fondazione Cariplo (grant n. 2011-0485; to Vittorio Ricci).

\section{Author details}

${ }^{1}$ Department of Molecular Medicine, Human Physiology Section, University of Pavia Medical School, Pavia, Italy. ${ }^{2}$ Department of Public Health, Hygiene Section, University of Naples "Federico II", Naples, Italy. ${ }^{3}$ Department of Translational Medical Sciences, University of Naples "Federico II", Naples, Italy. ${ }^{4}$ PhD School of Science, University of Basilicata, Potenza, Italy. ${ }^{5}$ Department of Clinical and Experimental Medicine, Chair of Gastroenterology, Second University of Naples, Naples, Italy. ${ }^{6}$ CEINGE Biotecnologie Avanzate, Naples, taly.

Received: 10 June 2014 Accepted: 19 August 2014

Published: 27 August 2014

\section{References}

1. Romano M, Ricci V, Zarrilli R: Mechanisms of disease: Helicobacter pylori-related gastric carcinogenesis-implications for chemoprevention. Nat Clin Pract Gastroenterol Hepatol 2006, 3:622-632.

2. Salama NR, Hartung ML, Müller A: Life in the human stomach: persistence strategies of the bacterial pathogen Helicobacter pylori. Nat Rev Microbiol 2013, 11:385-399.

3. Amieva MR, Vogelmann R, Covacci A, Tompkins LS, Nelson WJ, Falkow S: Disruption of the epithelial apical-junctional complex by Helicobacter pylori CagA. Science 2013, 300:1430-1434

4. Oldani A, Cormont M, Hofman V, Chiozzi V, Oregioni O, Canonici A, Sciullo A, Sommi P, Fabbri A, Ricci V, Boquet P: Helicobacter pylori counteracts the apoptotic action of its VacA toxin by injecting the CagA protein into gastric epithelial cells. PLoS Pathog 2009, 5:e1000603.

5. Olbermann P, Josenhans C, Moodley Y, Uhr M, Stamer C, Vauterin M, Suerbaum S, Achtman M, Linz B: A global overview of the genetic and functional diversity in the Helicobacter pylori cag pathogenicity island. PLoS Genet 2010, 6:e1001069.

6. Ricci V, Romano M, Bouquet P: Molecular cross-talk between Helicobacter pylori and human gastric mucosa. World J Gastroenterol 2011, 17:1383-1399.

7. Boquet $P$, Ricci V: Intoxication strategy of Helicobacter pylori VacA toxin. Trends Microbiol 2012, 20:165-174.

8. McGovern KJ, Blanchard TG, Gutierrez JA, Czinn SJ, Krakowka S, Youngman P: $Y$-Glutamyltransferase Is a Helicobacter pylori virulence factor but is not essential for colonization. Infect Immun 2001, 69:4168-4173.
9. Ricci V, Giannouli M, Romano M, Zarrilli R: Helicobacter pylori gamma-glutamyl transpeptidase and its pathogenic role. World J Gastroenterol 2014, 20:630-638.

10. Tomb JF, White O, Kerlavage AR, Clayton RA, Sutton GG, Fleischmann RD, Ketchum KA, Klenk HP, Gill S, Dougherty BA, Nelson K, Quackenbush J, Zhou L, Kirkness EF, Peterson S, Loftus B, Richardson D, Dodson R, Khalak HG, Glodek A, McKenney K, Fitzegerald LM, Lee N, Adams MD, Hickey EK, Berg DE, Gocayne JD, Utterback TR, Peterson JD, Kelley JM, et al: The complete genome sequence of the gastric pathogen Helicobacter pylori. Nature 1997, 88:539-554.

11. Alm RA, Ling L-SL, Moir DT, King BL, Brown ED, Doig PC, Smith DR, Noonan $B$, Guild BC, de Jonge BL, Carmel G, Tummino PJ, Caruso A, Uria-Nickelsen M, Mills DM, Ives C, Gibson R, Merberg D, Mills SD, Jiang Q, Taylor DE, Vovis GF, Trust TJ: Genomic sequence comparison of two unrelated isolates of the human gastric pathogen Helicobacter pylori. Nature 1999, 397:176-180.

12. Blanchard TG, Czinn SJ, Correa P, Nakazawa T, Keelan M, Morningstar L, Santana-Cruz I, Maroo A, McCracken C, Shefchek K, Daugherty S, Song Y, Fraser CM, Fricke WF: Genome sequences of 65 Helicobacter pylori strains isolated from asymptomatic individuals and patients with gastric cancer, peptic ulcer disease, or gastritis. Pathog Dis 2013, 68:39-43

13. Xia HH, Talley NJ: Apoptosis in gastric epithelium induced by Helicobacter pylori infection: implications in gastric carcinogenesis. Am J Gastroenterol 2001, 96:16-26.

14. Galgani M, Busiello I, Censini S, Zappacosta S, Racioppi L, Zarrilli R: Helicobacter pylori induces apoptosis of human monocytes, but not monocyte-derived dendritic cells: role of the cag pathogenicity island. Infect Immun 2004, 72:4480-4485.

15. Radin JN, González-Rivera C, Ivie SE, McClain MS, Cover TL: Helicobacter pylori VacA induces programmed necrosis in gastric epithelial cells. Infect Immun 2011, 79:2535-2543.

16. Lee A, O'Rourke J, De Ungria MC, Robertson B, Daskalopoulos G, Dixon MF: A standardized mouse model of Helicobacter pylori infection: introducing the Sydney strain. Gastroenterology 1997, 112:1386-1397.

17. Zhang S, Moss SF: Rodent models of Helicobacter infection, inflammation and disease. Methods Mol Biol 2012, 921:89-98.

18. Oertli M, Noben M, Engler DB, Semper RP, Reuter S, Maxeiner J, Gerhard M, Taube C, Müller A: Helicobacter pylori y-glutamyl transpeptidase and vacuolating cytotoxin promote gastric persistence and immune tolerance. Proc Natl Acad Sci U S A 2013, 110:3047-3052.

19. Steinert M, Leippe $M$, Roeder T: Surrogate host: protozoa and invertebrates for studying pathogen-host interactions. Int J Med Microbiol 2013, 293:321-332

20. Garclà-Lara J, Needham AJ, Foster SJ: Invertebrates as animal models for Staphylococcus aureus pathogenesis: a window into host-pathogen interaction. FEMS Immunol Med Microbiol 2005, 43:311-323.

21. Mylonakis E, Casadevall A, Ausubel FM: Exploiting amoeboid and nonvertebrate animal model systems to study the virulence of human pathogenic fungi. PLoS Pathog 2007, 3:e101.

22. Botham CM, Wandler AM, Guillemin K: A transgenic Drosophila model demonstrates that the Helicobacter pylori CagA protein functions as a eukaryotic Gab adaptor. PLoS Pathog 2008, 4:e1000064.

23. Wandler AM, Guillemin K: Transgenic expression of the Helicobacter pylori virulence factor CagA promotes apoptosis or tumorigenesis through JNK activation in Drosophila. PLoS Pathog 2012, 8:e1002939.

24. Bergin D, Reeves EP, Renwick J, Wientjes FB, Kavanagh K: Superoxide production in Galleria mellonella hemocytes: identification of proteins homologous to the NADPH oxidase complex of human neutrophils. Infect Immun 2005, 73:4161-4170.

25. Vogel H, Altincicek B, Glöckner G, Vilcinskas A: A comprehensive transcriptome and immune-gene repertoire of the lepidopteran model host Galleria mellonella. BMC Genomics 2011, 12:308.

26. Khoa DB, Takeda M: Expression analysis of inhibitor of apoptosis and related caspases in the midgut and silk gland of the greater wax moth, Galleria mellonella, during metamorphosis and under starvation. Gene 2012, 510:133-141.

27. Jander G, Rahme LG, Ausubel FM: Positive correlation between virulence of Pseudomonas aeruginosa mutants in mice and insects. J Bacterio/ 2000, 182:3843-3845. 
28. Peleg AY, Monga D, Pillai S, Mylonakis E, Moellering RC, Elioupoulos GM: Reduced susceptibility to vancomycin influences pathogenicity in Staphylococcus aureus infection. J Infect Dis 2009, 199:532-536.

29. Aperis G, Fuchs BB, Anderson CA, Warner JE, Calderwood SB, Mylonakis E: Galleria mellonella as a model host to study infection by the Francisella tularensis live vaccine strain. Microbes Infect 2007, 9:729-734.

30. Schell MA, Lipscomb L, DeShazer D: Comparative genomics and an insect model rapidly identify novel virulence genes of Burkholderia mallei. J Bacteriol 2008, 190:2306-2313.

31. Peleg AY, Jara S, Monga D, Eliopoulos GM, Moellering RC Jr, Mylonakis E: Galleria mellonella as a model system to study Acinetobacter baumannii pathogenesis and therapeutics. Antimicrob Agents Chemother 2009, 53:2605-2609.

32. Insua JL, Llobet E, Moranta D, Pérez-Gutiérrez C, Tomàs A, Garmendia J, Bengoechea JA: Modeling Klebsiella pneumoniae pathogenesis by infection of the wax moth Galleria mellonella. Infect Immun 2013, 81:3552.

33. Mylonakis E, Moreno R, El Khoury JB, Idnurm A, Heitman J, Calderwood SB, Ausubel FM, Diener A: Galleria mellonella as a model system to study Cryptococcus neoformans pathogenesis. Infect Immun 2005, 73:3842-3850

34. Brennan M, Thomas DY, Whiteway M, Kavanagh K: Correlation between virulence of Candida albicans mutants in mice and Galleria mellonella larvae. FEMS Immunol Med Microbiol 2002, 34:153-157.

35. Champion OL, Karlyshev AV, Senior NJ, Woodward M, La Ragione R, Howard SL, Wren BW, Titball RW: Insect infection model for Campylobacter jejuni reveals that $O$-methyl phosphoramidate has insecticidal activity. $J$ Infect Dis 2010, 201:776-782.

36. Senior NJ, Bagnall MC, Champion OL, Reynolds SE, La Ragione RM, Woodward MJ, Salguero FJ, Titball RW: Galleria mellonella as an infection model for Campylobacter jejuni virulence. J Med Microbiol 2011, 60:661-669.

37. Censini S, Lange C, Xiang Z, Crabtree JE, Ghiara P, Borodovsky M, Rappuoli R, Covacci A: cag, a pathogenicity island of Helicobacter pylori, encodes type I-specific and disease-associated virulence factors. Proc Natl Acad Sci US A 1996, 93:14648-14653.

38. Cover TL, Tummuru MK, Cao P, Thompson SA, Blaser MJ: Divergence of genetic sequences for the vacuolating cytotoxin among Helicobacter pylori strains. J Biol Chem 1994, 269:10566-10573.

39. Tummuru MK, Sharma SA, Blaser MJ: Helicobacter pylori picB, a homologue of the Bordetella pertussis toxin secretion protein, is required for induction of IL-8 in gastric epithelial cells. Mol Microbio/ 1995, 18:867-876.

40. Pèrez-Pèrez Gl, Olivares AZ, Cover TL, Blaser MJ: Characteristics of Helicobacter pylori variants selected for urease deficiency. Infect Immun 1992, 60:3658-3663.

41. Ricci V, Ciacci C, Zarrilli R, Sommi P, Tummuru MKR, Del Vecchio Blanco C, Bruni CB, Cover TL, Blaser MJ, Romano M: Effect of Helicobacter pylori on gastric epithelial cell migration and proliferation in vitro: role of VacA and CagA. Infect Immun 1996, 64:2829-2833.

42. Hofman V, Ricci V, Mograbi B, Brest P, Luciano F, Boquet P, Rossi B, Auberger P, Hofman P: Helicobacter pylori lipopolysaccharide hinders polymorphonuclear leukocyte apoptosis. Lab Invest 2001, 81:375-384

43. Cover TL, Hanson PI, Heuser JE: Acid-induced dissociation of VacA, the Helicobacter pylori cytotoxin, reveals its pattern of assembly. J Cell Biol 1997, 138:759-769.

44. Chiozzi V, Mazzini G, Oldani A, Sciullo A, Ventura U, Romano M, Boquet P, Ricci V: Relationship between VacA toxin and ammonia in Helicobacter pylori-induced apoptosis in human gastric epithelial cells. J Physiol Pharmacol 2009, 60:23-30.

45. Ricci V, Galmiche A, Doye A, Necchi V, Solcia E, Bouquet P: High cell sensitivity to Helicobacter pylori VacA toxin depends on a GPI-anchored protein and is not blocked by inhibition of the clathrin-mediated pathway of endocytosis. Mol Biol Cell 2000, 11:3897-3909.

46. van Engeland M, Nieland LJW, Ramaekers FCS, Schutte B, Reutelingsperger $C P$ : Annexin V-affinity assay: a review on an apoptosis detection system based on phosphatidylserine exposure. Cytometry 1998, 31:1-9.
47. Giannouli M, Antunes LCS, Marchetti V, Triassi M, Visca P, Zarrilli R: Virulence-related traits of epidemic Acinetobacter baumannii strains belonging to the international clonal lineages I-III and to the emerging genotypes ST25 and ST78. BMC Infect Dis 2013, 13:282.

48. Cover TL, Krishna US, Israel DA, Peek RM Jr: Induction of gastric epithelial cell apoptosis by Helicobacter pylori vacuolating cytotoxin. Cancer Res 2003, 63:951-957.

doi:10.1186/s12866-014-0228-0

Cite this article as: Giannouli et al:: Use of larvae of the wax moth Galleria mellonella as an in vivo model to study the virulence of Helicobacter pylori. BMC Microbiology 2014 14:228.

\section{Submit your next manuscript to BioMed Central and take full advantage of:}

- Convenient online submission

- Thorough peer review

- No space constraints or color figure charges

- Immediate publication on acceptance

- Inclusion in PubMed, CAS, Scopus and Google Scholar

- Research which is freely available for redistribution

Submit your manuscript at www.biomedcentral.com/submit
C) Biomed Central 